\section{RESISTENCIA A Phytophthora capsici LEO. DE CHILES NATIVOS DEL SUR DE PUEBLA, MÉXICO}

\section{RESISTANCE TO Phytophthora capsici LEO. OF CHILLI PEPPER LANDRACES OF SOUTHERN PUEBLA, MÉXICO}

\author{
Sara H. Morán-Bañuelos ${ }^{1 *}$, Víctor H. Aguilar- \\ Rincón $^{1}$, Tarsicio Corona-Torres ${ }^{1}$ y Emma \\ Zavaleta-Mejía
}

\begin{abstract}
${ }^{1}$ Postgrado de Recursos Genéticos y Productividad y ${ }^{2}$ Postgrado de Fitosanidad, Colegio de Postgraduados-Campus Montecillo. Km. 36.5 Carr. México-Texcoco. 56230, Montecillo, Texcoco, Estado de México. Tel. (595) 952-02-00 Ext. 1588 y Fax: (595) 952-02-62.

* Autor para correspondencia (shiran@colpos.mx)

\section{RESUMEN}

Las pérdidas en la producción de chile (Capsicum spp.) ocasionadas por infección con Phytophthora capsici LEO. han llegado a ser considerables, por lo que se ha planteado enfrentar el problema fitosanitario mediante la búsqueda de resistencia genética en las poblaciones de chile nativo. Se evaluó bajo condiciones de invernadero la resistencia a $P$. capsici de 29 poblaciones de chile colectadas en el sur del Estado de Puebla. Se registraron las variables: aparición de síntomas, incidencia y acumulación de necrosis en la base del tallo, $\mathbf{y}$ aspecto general de la planta; además, se obtuvo el área bajo la curva del progreso de la enfermedad tanto en incidencia como en severidad. Los análisis de varianza y comparación de medias mostraron diferencias significativas entre poblaciones, lo cual indica que algunos materiales presentan mecanismos de defensa particulares que podrían ser explorados y aprovechados en programas de mejoramiento.
\end{abstract}

Palabras clave: Capsicum annuum, marchitez, resistencia genética, secadera del chile.

\section{SUMMARY}

Pepper production (Capsicum spp.) is severely affected by infection of Phytophthora capsici LEO. The search for genetic resistance in native pepper populations is considered an alternative to solve this phytosanitary problem. The resistance to Phytophthora capsici of 29 accessions collected in the State of Puebla, México was evaluated in greenhouse experiments. Appearance of symptoms, incidence, accumulated necrosis in the base of the stem, and the disease progress curve were registered as response variable. Variance analysis and means comparisons showed significant differences between populations, thus indicating that some materials have particular defense mechanisms that could be explored and used in plant breeding protocols.

Index words: Capsicum annuum, genetic resistance, pepper root rot, pepper wilt.

\section{INTRODUCCIÓN}

La planta de chile (Capsicum spp.) es afectada por la enfermedad conocida como marchitez o secadera, que se encuentra presente en todo el mundo y es producida por el complejo de patógenos Phytophthora spp., Fusarium spp., Pythium spp. y Rhizoctonia solani (Velásquez et al., 2001). De este complejo, la infección causada por $P$. capsici es particularmente importante porque la planta atacada se vuelve vulnerable a ser invadida por otros patógenos (Erwin y Ribeiro, 1996; Hausbeck y Lamour, 2004).

El oomiceto se encuentra en el suelo y desde ahí sus propágulos son capaces de diseminarse en corrientes de agua e infectar las raíces o la base del tallo, tanto en plántula como en etapa adulta; en la raíz genera pudrición y en la base del tallo un estrangulamiento que bloquea el xilema e interrumpe el paso del agua, causa amarillamiento de las hojas, favorece la incidencia del tizón de hojas ocasionado por Phytophthora spp., defoliación rápida, pudrición de frutos y muerte de la planta (Velásquez et al., 2001).

Las pérdidas en la producción pueden llegar a ser considerables y el control químico y cultural no ha sido suficiente para controlar el problema (Rincón y Velásquez, 1999). Diversos autores consideran que la resistencia genética es una alternativa sustentable para enfrentar el problema fitosanitario y a la vez reducir el uso de fungicidas y la degradación del suelo (Ogundiwin et al., 2005). Al respecto, las poblaciones nativas representan un reservorio de genes de resistencia a diversas enfermedades que debe ser explorado para identificar germoplasma como fuente de resistencia, como en los chiles criollos de Morelos donde se ha detectado resistencia, la cual se ha incorporado a materiales mejorados (Laborde y Pozo, 1984).

En el Estado de Puebla se ha encontrado diversidad genética en materiales locales o nativos representada en los tipos: Ancho, Mulato, Miahuateco, Copi, Loco, criollos de Tecomatlán, Serranito $(C$. annuum L.) y Manzano (C. pubescens Jacq.) (Aguilar et al., 2006). En los últimos años la alta incidencia de enfermedades del suelo ha provocado que el cultivo del chile Ancho, predominante en Puebla, se haya desplazado hacia otras áreas, como a la región Centro-Norte del país que ocupa 
el primer lugar en producción (Chávez et al., 1995; Velásquez et al., 2003; González et al., 2004).

El objetivo del presente trabajo fue estimar el grado de resistencia a $P$. capsici que poseen poblaciones de chile nativo del sur del Estado de Puebla, en los tipos: Miahuateco, Copi y criollos de Tecomatlán, así como detectar germoplasma con potencial como fuente de resistencia genética para su posterior incorporación a un programa de mejoramiento de esta especie.

\section{MATERIALES Y MÉTODOS}

Durante el ciclo agrícola primavera-verano 2004 se exploraron ocho municipios del sur del Estado de Puebla, en las que se colectaron 29 poblaciones de chile nativo denominados regionalmente como Miahuateco, Copi y de Tecomatlán (Amarillo, Colorado y Negro) (Figura 1). En cada población se cosecharon los frutos de 20 a 40 plantas aparentemente sanas, de una parcela agrícola sometida a la forma tradicional de cultivo. En agosto del año 2005 se estableció un ensayo en invernadero en un diseño completamente al azar con dos repeticiones, donde los tratamientos estuvieron constituidos por las 29 poblaciones colectadas y donde la unidad experimental fue de 10 a 30 plantas. Después de la siembra en almácigo, cuando las plántulas tenían una altura aproximada de $15 \mathrm{~cm}$, se trasplantaron a vasos de poliestireno de $1 \mathrm{~L}$ de capacidad; como sustrato se usó una mezcla 3:1 de suelo rico en materia orgánica previamente tratado bajo presión de vapor durante $2 \mathrm{~h}$ y "peat moss".

Como fuente de inóculo se utilizó el aislamiento 6143 de $P$. capsici altamente virulento proporcionado por la Universidad Estatal de Nuevo México, en EE. UU. El oomiceto se cultivó in vitro en medio esterilizado de agarjugo V8® a $29{ }^{\circ} \mathrm{C}$ durante $20 \mathrm{~d}$; enseguida se sumergió en agua destilada estéril y se mantuvo a temperatura ambiente para promover la generación de esporangios. Siete días después se verificó la formación de esporangios y éstos se incubaron a $8{ }^{\circ} \mathrm{C}$ para inducir la liberación de zoosporas al medio acuoso, lo cual se monitoreó cada 15 min; una vez que se observó la liberación de zoosporas, en un recipiente de vidrio se colectó el medio acuoso contenido en cada caja, se homogeneizó y se cuantificó el número de zoosporas por $\mathrm{mL}$ con ayuda de un hematocitómetro Neubauer-imp ${ }^{\circledR}$ (Marienfeld, Alemania).

Cuando las plantas exhibían ocho pares de hojas, cada una se inoculó con $2 \mathrm{~mL}$ de una suspensión de 50000 zoosporas por $\mathrm{mL}$. La suspensión se inyectó en el suelo cerca del tallo a una profundidad de $2 \mathrm{~cm}$ aproximadamente. Después de la inoculación $(1.5 \mathrm{~h})$, el suelo de cada maceta se regó a saturación con el propósito de crear un microambiente con alta humedad que facilitara la dispersión del inóculo y la infección de las plantas.

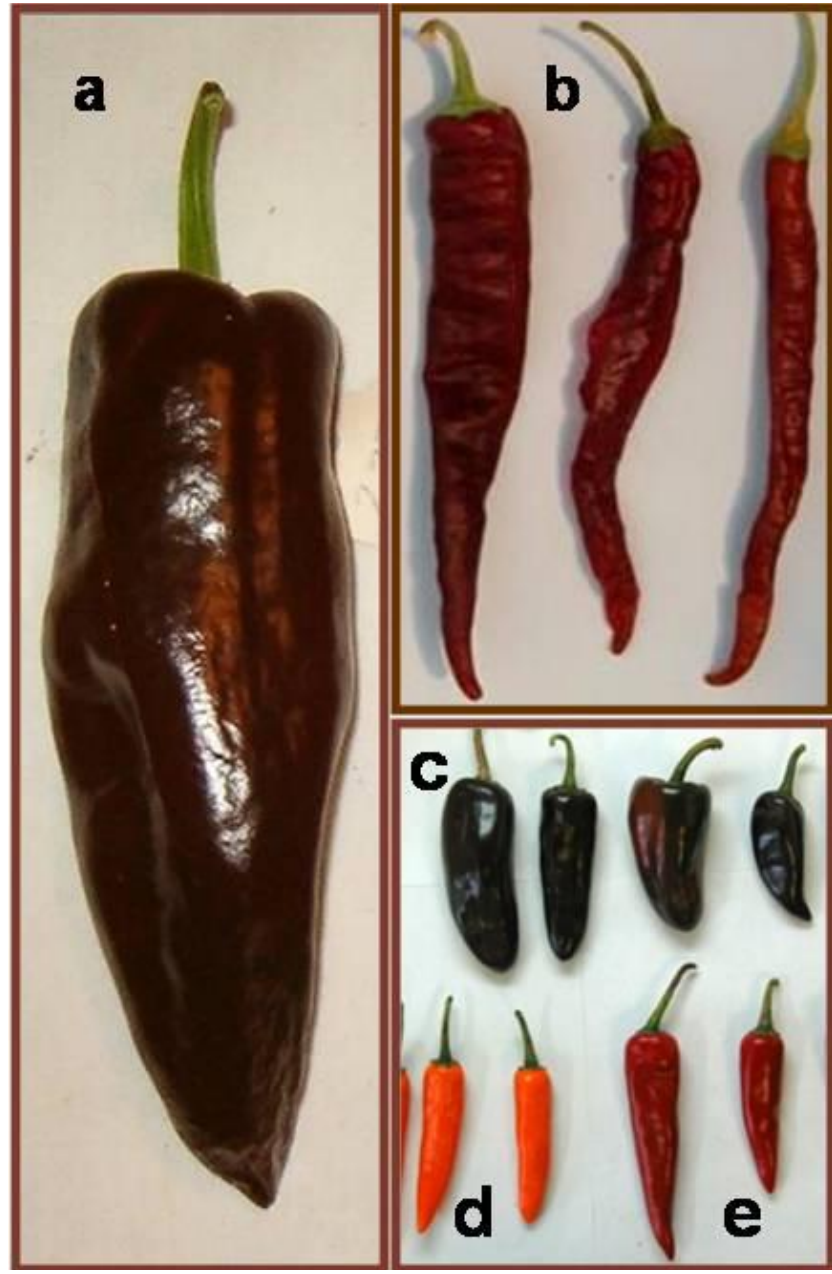

FIGURA 1. Frutos representativos de los tipos de chile reconocidos localmente como: Miahuateco (a), Copi (b), Criollo negro de Tecomatlán (c), Criollo amarillo de Tecomatlán (d) y Criollo colorado de Tecomatlán (e).

Los síntomas de la enfermedad se cuantificaron a partir del cuarto día después de la inoculación (dpi) y cada 48 h para un total de 12 registros, mediante las siguientes variables: incidencia $(\mathrm{Y})$, representada como el porcentaje de plantas en cada unidad experimental con necrosis en la base del tallo; longitud de necrosis en el tallo $(\mathrm{N})$, medida en $\mathrm{cm}$ a partir del nivel del suelo; y severidad de la enfermedad $(S)$ con base en una escala con categorías nominales arbitrarias del aspecto general de la planta: 0, hojas y tallos turgentes; 3 , hojas inferiores con pérdida de turgencia solamente; 5, hojas medias e inferiores marchitas y la punta del tallo con inclinación ligera; 7, hojas marchitas y punta del tallo colgante; 9, planta muerta. Los días posteriores a la inoculación (dpi) requeridos para la acumulación de $50 \%$ de incidencia se reportaron como DIn. 
La incidencia máxima de la enfermedad (Ymax) se calculó como el porcentaje de plantas enfermas por población, alcanzado al final de la prueba ( $26 \mathrm{dpi})$. Se registró también el tiempo requerido por cada unidad experimental para alcanzar la incidencia máxima (DYmax) y la necrosis final (Nf) representada por la longitud del tallo con necrosis, medida al final de la prueba. Se obtuvo el área bajo la curva del progreso de la enfermedad (ABCPE) por el método de integración trapezoidal (Campbell y Madden, 1990) para las variables Y (ABCPEi) y $\mathrm{S}$ (ABCPEs).

Con los datos obtenidos se hicieron análisis de varianza y comparación de medias (Tukey, 0.05) entre poblaciones, con el programa computacional SAS (SAS Institute, 1999).

\section{RESULTADOS Y DISCUSIÓN}

Todas las poblaciones inoculadas con $P$. capsici mostraron síntomas de marchitez y desarrollaron necrosis en la base del tallo en más de $60 \%$ de sus plantas; sin embargo, el avance de la enfermedad mostró una respuesta diferencial entre los materiales nativos evaluados, diferencial que se atribuye a variación genética en la resistencia a enfermedades. El tiempo promedio requerido para la aparición de necrosis en $50 \%$ de las plantas (DIn) fue de $10 \mathrm{dpi}$, pero en las poblaciones Miahuateco excepto en CP645, la enfermedad se estableció en un tiempo significativamente más corto con respecto al chile colorado de Tecomatlán (19 dpi); en ésta última se observó un retraso en el avance del oomiceto durante las primeras etapas de infección, lo cual sugiere la posible presencia de uno o varios mecanismos de defensa para contrarrestar el progreso de la enfermedad.

Seis poblaciones tuvieron valores de 60 a $80 \%$ para Ymax, cuatro de ellas del tipo Miahuateco provenientes de Tepanco (CP631) y Tlacotepec (CP642, CP645 y CP646), y las otras dos son de Tecomatlán (CP661a y CP661c). El resto de las poblaciones mostraron Ymax entre 80 y $100 \%$, significativamente superiores a los tipos Amarillo y Colorado en varios casos (Cuadro 1). Los materiales que alcanzaron una Ymax de $100 \%$ fueron CP644, CP647, CP650, CP657, CP654c у CP656, que podrían ser utilizados como testigos susceptibles en evaluaciones posteriores.

Las poblaciones del tipo Miahuateco CP657 y CP646 alcanzaron la incidencia máxima en un tiempo significativamente menor que el Miahuateco CP639, que el CP651 del tipo Copi proveniente de Yehualtepec, y que los chiles de Tecomatlán, que registraron el máximo número de días para alcanzar la incidencia máxima (26 dpi). Respecto a la necrosis final en el tallo (Nf), ésta fue de $5.4 \mathrm{~cm}$ en promedio para todas las colectas; los valores mínimos se registraron en CP661c, CP646, CP661a y CP645, pero sólo en las poblaciones CP646, CP661a y CP661c los periodos de incubación largos estuvieron relacionados con baja incidencia y menor acumulación de necrosis final. Es posible que durante estos largos periodos de incubación, las plantas hayan podido expresar uno o más mecanismos de defensa para contrarrestar la proliferación del oomiceto en los tejidos de conducción (Velásquez et al., 2002).

Resultados semejantes sobre la incidencia han sido encontrados al evaluar la enfermedad en una localidad con presencia abundante del patógeno en el suelo, mientras que Chávez et al. (1995) y Yánez et al. (2001) reportaron incidencias en campo por debajo de $70 \%$. En la presente investigación sólo en CP661a y CP661c se registraron valores cercanos a aquellos previamente reportados, debidos posiblemente a que las condiciones en que se desarrolló el experimento favorecieron la infección y desarrollo del patógeno. No obstante que en otras investígaciones como las de Bosland y Lindsey (1991) y Sy et al. (2008) han utilizado concentraciones de 10000 zoosporas, la alta densidad de inóculo aplicada en el presente trabajo pudo haber reducido la posibilidad de escape a la enfermedad y permitir así la expresión de la resistencia, como sugieren Andrés et al. (2005) hacer en los programas de mejoramiento.

El análisis de varianza del ABCPEi y ABCPEs mostró diferencias significativas en la respuesta de las poblaciones a la infección y el efecto sobre el aspecto general de la planta (Cuadro 2), ya que el chile criollo de Tecomatlán CP661c tuvo valores menores $(\mathrm{P} \leq 0.05)$ que algunas poblaciones de Miahuateco y Copi; las poblaciones con la mínima $\mathrm{ABCPEi}$ en cada tipo fueron: CP645 en el tipo Miahuateco, CP651 en Copi, y CP661c de Tecomatlán.

De manera general, valores bajos de ABCPEi estuvieron asociados a valores altos de DIn y DYmax. En particular, los valores en ABCPEi para CP661a y CP661c (864.2 y 763.2, respectivamente) fueron menores que lo reportado por Chávez et al. (1995) para sus mejores tratamientos de control de marchitez en chile criollo Cuayucatepec (993.5) considerado como tolerante para la región de Valsequillo, Puebla. Al considerar que la escala de severidad indica de manera indirecta el grado de la infección por el oomiceto y los efectos que tiene en el aspecto general de la planta, los valores bajos de ABCPEs podrían sugerir mecanismos de defensa particulares que permiten contrarrestar el efecto de la invasión de haces vasculares sobre los procesos fisiológicos que determinan el vigor de la planta. Así, en los chiles de Tecomatlán 
donde la infección produjo una disminución en el vigor, éstos tuvieron una reacción de resistencia intermedia ya que retrasaron el establecimiento de la enfermedad, la proliferación del patógeno y el avance del daño o necrosis, con una diferencia notable al ser comparadas con las susceptibles de Tlacotepec, Yehualtepec y Tecamachalco.

Los posibles mecanismos de defensa que intervinieron en algunas de las poblaciones analizadas podrían estar determinados por contenidos genéticos específicos, dada la naturaleza poligénica de la resistencia a $P$. capsici (Ogundiwin et al., 2005). Los tipos Miahuatecos y Copi fueron considerados por Romero (1962) dentro de los tipos susceptibles; no obstante, la importancia de éstos a nivel regional hace imperante el generar información respecto a la resistencia/susceptibilidad del recurso genético nativo y ampliar su evaluación con aislamientos procedentes de las zonas de estudio, ya que una limitante para obtener variedades resistentes a $P$. capsici es la variabilidad patogénica del oomiceto (Fernández et al., 2004). Lo anterior indica la necesidad de ampliar el estudio sobre la genética de la resistencia para detectar genes, seleccionar e incorporar germoplasma sobresaliente a un protocolo de mejoramiento genético.

Cuadro 1. Comportamiento promedio de poblaciones de chile (C. annuum L.) para las variables indicadoras de la marchitez al inocularse con $P$. capsici.

\begin{tabular}{|c|c|c|c|c|c|c|c|c|c|c|}
\hline \multirow{2}{*}{$\begin{array}{r}\text { Tipo } \\
\text { Miahuateco }\end{array}$} & \multirow{2}{*}{$\begin{array}{l}\text { Municipio } \\
\text { Tepanco }\end{array}$} & \multirow{2}{*}{$\begin{array}{c}\text { Población } \\
\text { CP631 }\end{array}$} & \multicolumn{2}{|c|}{ DIn } & \multicolumn{2}{|c|}{$Y \max$} & \multicolumn{2}{|c|}{ DYmax } & \multicolumn{2}{|c|}{$\mathrm{Nf}$} \\
\hline & & & 9 & $\mathrm{c}$ & 76.2 & abcd & 20 & $a b c$ & 5.3 & abcd \\
\hline & & CP632 & 9 & $\mathrm{c}$ & 98.2 & $a b$ & 23 & $\mathrm{ab}$ & 5.6 & abcd \\
\hline & & CP633 & 10 & $\mathrm{bc}$ & 89.9 & abcd & 20 & $a b c$ & 5.0 & abcd \\
\hline & & СР634 & 9 & $\mathrm{c}$ & 87.0 & abcd & 23 & $a b$ & 5.3 & abcd \\
\hline & & CP635 & 8 & $\mathrm{c}$ & 93.0 & $a b c$ & 23 & $a b$ & 6.1 & abcd \\
\hline & Tehuacán & CP637 & 8 & $\mathrm{c}$ & 91.3 & abcd & 14 & $a b c$ & 6.4 & abcd \\
\hline & & СР639 & 9 & c & 94.4 & $a b$ & 26 & $\mathrm{a}$ & 7.3 & $\mathrm{a}$ \\
\hline & Miahuatlán & СР640 & 9 & $\mathrm{bc}$ & 85.2 & abcd & 19 & $a b c$ & 6.8 & $a b c$ \\
\hline & & CP641 & 9 & $\mathrm{c}$ & 83.0 & abcd & 23 & $\mathrm{ab}$ & 5.9 & abcd \\
\hline & Tlacotepec & CP642 & 10 & $\mathrm{bc}$ & 76.1 & abcd & 23 & $a b$ & 4.6 & abcd \\
\hline & & СР643 & 10 & $\mathrm{bc}$ & 95.4 & $\mathrm{ab}$ & 23 & $a b$ & 5.4 & abcd \\
\hline & & CP644 & 10 & $\mathrm{bc}$ & 100.0 & $\mathrm{a}$ & 20 & $a b c$ & 5.1 & abcd \\
\hline & & CP645 & 13 & $a b c$ & 70.6 & bcd & 20 & $a b c$ & 3.5 & abcd \\
\hline & & CP646 & 11 & $\mathrm{bc}$ & 75.0 & abcd & 12 & $\mathrm{bc}$ & 2.9 & $\mathrm{~cd}$ \\
\hline & & CP647 & 9 & $\mathrm{c}$ & 100.0 & $\mathrm{a}$ & 17 & $a b c$ & 6.9 & $a b$ \\
\hline & & CР648 & 11 & $\mathrm{bc}$ & 93.7 & $a b c$ & 20 & $a b c$ & 4.5 & abcd \\
\hline & Yehualtepec & СР649 & 10 & $\mathrm{bc}$ & 93.7 & $a b c$ & 23 & $a b$ & 5.4 & abcd \\
\hline & & CP650 & 9 & $\mathrm{c}$ & 100.0 & $\mathrm{a}$ & 17 & $a b c$ & 7.2 & $a b$ \\
\hline & & CP652 & 11 & $\mathrm{bc}$ & 83.3 & abcd & 17 & $a b c$ & 5.5 & abcd \\
\hline & & CP653 & 10 & $\mathrm{bc}$ & 94.2 & $a b c$ & 20 & $a b c$ & 5.5 & abcd \\
\hline & Xochitlán & CP654 & 9 & $\mathrm{bc}$ & 95.4 & $\mathrm{ab}$ & 20 & $a b c$ & 6.0 & abcd \\
\hline & & CP655 & 10 & $\mathrm{bc}$ & 95.4 & $\mathrm{ab}$ & 19 & $a b c$ & 6.6 & abcd \\
\hline & Tecamachalco & CP657 & 8 & $\mathrm{c}$ & 100.0 & $\mathrm{a}$ & 9 & $\mathrm{c}$ & 6.5 & abcd \\
\hline \multirow[t]{3}{*}{ Copi } & Yehualtepec & CP651 & 10 & $\mathrm{bc}$ & 89.2 & abcd & 26 & $\mathrm{a}$ & 5.4 & abcd \\
\hline & Xochitlán & CP654c & 9 & $\mathrm{c}$ & 100.0 & $\mathrm{a}$ & 15 & $a b c$ & 5.0 & abcd \\
\hline & Tecamachalco & СР656 & 10 & $\mathrm{bc}$ & 100.0 & $\mathrm{a}$ & 14 & $a b c$ & 6.1 & abcd \\
\hline Criollos de & Tecomatlán & CP661a & 16 & $a b$ & 63.8 & d & 26 & $\mathrm{a}$ & 3.4 & bcd \\
\hline \multirow[t]{3}{*}{ Tecomatlán } & Tecomatlán & CP661c & 19 & $\mathrm{a}$ & 65.3 & $\mathrm{~cd}$ & 26 & $\mathrm{a}$ & 2.8 & $\mathrm{~d}$ \\
\hline & Tecomatlán & CP661n & 12 & $\mathrm{bc}$ & 82.6 & abcd & 26 & $\mathrm{a}$ & 4.7 & abcd \\
\hline & \multicolumn{2}{|c|}{ Media } & \multicolumn{2}{|l|}{10} & \multicolumn{2}{|l|}{89.0} & \multicolumn{2}{|l|}{20} & \multicolumn{2}{|l|}{5.4} \\
\hline
\end{tabular}

DIn = días transcurridos desde la inoculación hasta la aparición necrosis en $50 \%$ de las plantas. Ymax $=$ incidencia máxima $(\%)$; DYmax $=$ días requeridos para alcanzar la máxima incidencia; $\mathrm{Nf}=$ necrosis final en la base del tallo $(\mathrm{cm})$; Medias con letras iguales en una columna, no son estadísticamente diferentes (Tukey, 0.05). $\uparrow$ CP661a $=$ amarillo; CP661c $=$ colorado y CP661n $=$ negro. 
Cuadro 2. Comparación de medias para el Área bajo la curva del progreso de la enfermedad a partir de los datos de incidencia (ABCPEi) y severidad (ABCPEs) en poblaciones de chile (C. annuum $\mathrm{L}$.) al inocularse con $P$. capsici.

\begin{tabular}{|c|c|c|c|c|}
\hline Tipo & Municipio & Población & ABCPEi (\%/d) & ABCPEs (calificación/d) \\
\hline \multirow[t]{23}{*}{ Miahuateco } & Tepanco & CP631 & 1252.4 abcd & $84.0 \quad \mathrm{ab}$ \\
\hline & & CP632 & 1554.9 abcd & $81.0 \mathrm{ab}$ \\
\hline & & CP633 & $1466.2 \mathrm{abcd}$ & $93.0 \mathrm{ab}$ \\
\hline & & СР634 & 1422.4 abcd & $96.0 \mathrm{ab}$ \\
\hline & & CP635 & $1613.1 \mathrm{abc}$ & $99.0 \mathrm{a}$ \\
\hline & Tehuacán & CP637 & $1617.0 \mathrm{abc}$ & $93.0 \mathrm{ab}$ \\
\hline & & CP639 & 1501.9 abcd & $93.0 \mathrm{ab}$ \\
\hline & Miahuatlán & СР640 & $1422.2 \mathrm{abcd}$ & $87.0 \mathrm{ab}$ \\
\hline & & CP641 & 1437.0 abcd & $87.0 \mathrm{ab}$ \\
\hline & Tlacotepec & CP642 & $1187.2 \mathrm{abcd}$ & $87.0 \mathrm{ab}$ \\
\hline & & CP643 & $1452.9 \mathrm{abcd}$ & $87.0 \mathrm{ab}$ \\
\hline & & CP644 & $1521.3 \mathrm{abcd}$ & $93.0 \mathrm{ab}$ \\
\hline & & CP645 & 999.8 bcd & $75.0 \mathrm{ab}$ \\
\hline & & CP646 & 1190.9 abcd & $79.0 \mathrm{ab}$ \\
\hline & & CP647 & $1713.2 \mathrm{ab}$ & $87.0 \mathrm{ab}$ \\
\hline & & CP648 & $1473.7 \mathrm{abcd}$ & $99.0 \mathrm{a}$ \\
\hline & Yehualtepec & CP649 & 1331.1 abcd & 87.0 \\
\hline & & CP650 & $1715.6 \mathrm{ab}$ & $87.0 \mathrm{ab}$ \\
\hline & & CP652 & $1360.2 \mathrm{abcd}$ & $99.0 \quad \mathrm{a}$ \\
\hline & & СР653 & 1497.9 abcd & 87.0 \\
\hline & Xochitlán & CP654 & $1486.2 \mathrm{abcd}$ & $91.0 \mathrm{ab}$ \\
\hline & & CP655 & 1539.0 abcd & $103.0 \mathrm{a}$ \\
\hline & Tecamachalco & CP657 & $1848.2 \mathrm{a}$ & $102.0 \mathrm{a}$ \\
\hline \multirow[t]{3}{*}{ Copi } & Yehualtepec & CP651 & 1391.7 abcd & 81.0 \\
\hline & Xochitlán & CP654c & $1648.7 \mathrm{abc}$ & 87.0 \\
\hline & Tecamachalco & CP656 & $1590.8 \mathrm{abcd}$ & 87.0 \\
\hline Criollos de & Tecomatlán & CP661a & $864.2 \mathrm{~cd}$ & 78.0 \\
\hline \multirow[t]{3}{*}{ Tecomatlán } & Tecomatlán & CP661c & $763.2 \mathrm{~d}$ & 63.0 \\
\hline & Tecomatlán & CP661n & $1156.3 \mathrm{abcd}$ & $87.0 \mathrm{ab}$ \\
\hline & & Media & 1414.5 & 88.2 \\
\hline
\end{tabular}

Medias con letras iguales no son estadísticamente diferentes (Tukey, 0.05). CP661a $=$ amarillo; CP661c $=$ colorado y CP661n $=$ negro.

\section{CONCLUSIONES}

Los resultados obtenidos en los parámetros registrados muestran que, a pesar del severo daño causado por la inoculación con $P$. capsici, la variación entre plantas dentro de la población, entre poblaciones y entre tipos, permitió identificar a las colectas de chile del municipio de Tecomatlán como fuente de germoplasma potencial con resistencia genética a $P$. capsici.

\section{BIBLIOGRAFÍA}

Andrés A J L, A Rivera M, J Fernández P (2005) Resistance of pepper germplasm to Phytophthora capsici isolates collected in northwest Spain. Spanish. J. Agric. Res. 3:429-436.

Aguilar R V H, T Corona T, S H Morán B (2006) Chiles nativos (Capsicum spp., Solanaceae) de los estados de Puebla y Morelos. In: Avances de Investigación de la Red de Hortalizas del SINAREFI. P López L, S Montes H (eds). Libro Científico Núm. 1. Campo Experimental Bajío INIFAP. Celaya, Guanajuato, México. pp:28-58.
Bosland P W, D L Lindsey (1991) A seedling screen for Phytophthora root rot of pepper, Capsicum annuum. Plant Dis. 75:10481050.

Campbell C L, L V Madden (1990) Introduction to Plant Disease Epidemiology. John Wiley and Sons, New York. 532 p.

Chávez A J J, E Zavaleta M, D Téliz O (1995) Control integrado de la marchitez del chile (Capsicum annum L.) ocasionado por el hongo Phytophthora capsici L. en la región de Valsequillo, Puebla, México. Fitopatología 30:47-55.

Erwin C D, K O Ribeiro (1996) Phytophthora Diseases Worldwide. APS - Press. U.S.A. 562 p.

Fernández P S P, C L Biles, M E Waugh, K Onsurez-Waugh, G Rodríguez A, C M Liddell (2004) Characterization of southern New Mexico Phytophtora capsici Leonian isolates from pepper (Capsicum annuum L.). Rev. Mex. Fitopatol. 22:82-89.

González P E, M J Yáñez M, V Santiago S, A Montero P (2004) Biodiversidad fungosa en la marchitez del chile y algunos factores involucrados, en Tlacotepec de José Manzo, El Verde, Puebla. Agrociencia 38:653-661.

Hausbeck M K, K H Lamour (2004) Phytophthora capsici on vegetable crops: research progress and management challenges. Plant Dis. 88:1292-1303.

Laborde C J A, O Pozo C (1984) Presente y Pasado del Chile en México. Secretaría de Agricultura y Recursos Hidráulicos. Publicación Especial No. 85. Instituto Nacional de Investigaciones Agrícolas (SARH-INIA), México. 80 p. 
Ogundiwin E A, T F Berke, M Massoudi, L L Black, G Huestis, D Choi, S Lee, J P Prince (2005) Construction of 2 intraspecific linkage maps and identification of resistance QTL's for Phytophthora capsici root-rot and foliar blight diseases of pepper (Capsicum annuum L.). Genome 48:698-711.

Rincón V J F, R Velásquez V (1999) Reacción de genotipos de chile (Capsicum annuum L.) a pudriciones radicales en Zacatecas. Hort. Mex. 7:130.

Romero C S (1962) Inoculación artificial de chile en el campo con Phytophthora capsici Leo. Agric. Téc. Méx. 2:79-80.

SAS Institute (1999) SAS/STAT. User's Guide. Version 8, Vol. 1-5. SAS Publishing. Cary, N.C. 3848 p.

Sy O, R Steiner, P W Bosland (2008) Recombinant inbred line differential identifies race-specific resistance to Phytophthora root rot in Capsicum annuum. Phytopatology 98:867-870.
Velásquez V R M, M M Medina A, J J Luna R (2001) Sintomatología y géneros de patógenos asociados con las pudriciones de la raíz del chile (Capsicum annuиm L.) en el Norte-Centro de México. Rev. Mex. Fitopatol. 19:175-181.

Velásquez V R M, M M Medina A, L M Macías V (2003) Reacción de líneas avanzadas de chile (Capsicum annuum L.) provenientes de Zacatecas a enfermedades comunes en Aguascalientes, México. Rev. Mex. Fitopatol. 21:71-74.

Yáñez J G M, E Zavaleta M, C Flores R, J Chávez A, R Valdivia A (2001) Management of wilting ( $P$. capsici Leo.), root galling ( $N$. aberrans Thorne and Allen), and virosis in pepper ( $C$. annuит L.). Rev. Mex. Fitopatol. 19: 40-48. 